\title{
Three-dimensional regulation of HOXA cluster genes by a cis-element in hematopoietic stem cell and leukemia.
}

Xue Qing David Wang ${ }^{1}$, Haley Gore ${ }^{1}$, Pamela Himadewi ${ }^{1}$, Fan Feng ${ }^{2}$, Lu Yang ${ }^{3}$, Wanding

Zhou ${ }^{1,5}$, Yushuai Liu' ${ }^{1}$, Xinyu Wang ${ }^{4}$, Chun-wei Chen ${ }^{3}$, Jianzhong Su ${ }^{4}$, Jie Liu' ${ }^{2}$, Gerd Pfeifer ${ }^{1, *}$, Xiaotian Zhang,

1. Center for Epigenetics, Van Andel Research Institute, Grand Rapids, Michigan

2. Department of Computational Medicine and Bioinformatics, University of Michigan, Ann Arbor, Michigan.

3. Department of System Biology, City of Hope Cancer Center, California

4. Institute of Biomedical Big Data, Wenzhou Medical University, Wenzhou, China

5. Current address: Children Hospital of Philadelphia, University of Pennsylvania

*. These authors jointly supervise this project

Correspondence should be addressed to Xiaotian Zhang: zxtzhangqian@gmail.com 


\begin{abstract}
:
Proper gene regulation is crucial for cellular differentiation, and dysregulation of key genes can lead to diseased states such as cancer. The HOX transcription factors play such a role during hematopoiesis, and aberrant expression of certain HOXA genes is found in certain acute myeloid leukemias (AMLs). While studies have shown that these genes are targeted by a variety of mutant proteins including mutant NPM1, MLL fusions, and NUP98 fusions, little is known about how long-range 3D chromatin interactions regulate the HOXA genes in normal hematopoiesis and leukemia. Here, we report the interaction between the HOXA cluster with a $\sim 1.3 \mathrm{Mb}$ upstream DNA methylation Canyon termed "Geneless Canyon" (GLC) in human CD34+/CD38- hematopoietic stem cells (HSCs) and AML cell lines. We show that CRISPRCas9 mediated deletion of the whole GLC region reduces the expression of the distal HOXA genes and compromises HSC and leukemia cells self-renewal. This long-range chromatin interaction brings the HOXA cluster in contact with the nuclear pore complex (NPC) at the nuclear periphery, which promotes HOXA gene expression and maintains HSC and leukemia cell self-renewal. These findings reveal how long-range 3D chromatin organization regulates key transcription factor genes in both normal and diseased hematopoietic cells.
\end{abstract}




\section{Main Text:}

Hematopoietic stem cells (HSCs) give rise to a wide variety of blood cells through a tightly regulated differentiation process, in which the $H O X$ transcription factors play a key role ${ }^{1,2}$. Several HOXA genes have been shown to maintain a high level of stem cell self-renewal at the early stages of hematopoiesis, but these genes are repressed as the cells differentiate into specific lineages ${ }^{3}$. Overexpression of these HOXA genes is found in several acute myeloid leukemias (AML), where the genes are often directly targeted by chimeric proteins such as MLLfusions and NUP98-fusion ${ }^{4,5}$. Recent studies in mouse limb development have identified several enhancers interacting with the HOXA locus, contributing to their gene regulation during development ${ }^{6,7}$. As of yet, little is known about the role of $3 \mathrm{D}$ chromatin organization during hematopoiesis and whether distant enhancers are involved in the regulation of the HOXA genes in both normal and diseased states.

The HOXA gene cluster is located between two topologically associating domains (TADs) and acts as a boundary for both 5' and 3' TADs. At the 5' end (centromeric), there is a $\sim 1 \mathrm{Mb}$ TAD encompassing the HIBADH, TAX1BP1 and JAZF1 genes, while the 3' (telomeric) TAD spans $\sim 1.3 \mathrm{Mb}$ and includes several subTADs formed around genes (Figure 1a). We previously identified an interaction between the HOXA locus and an upstream DNA methylation canyon, designated Geneless Canyon or GLC, in cord blood CD34+ HSPCs ${ }^{8}$. This long-range interaction anchors the 3' TAD despite a lack of convergent CTCF binding sites (Supplemental Figure S4). Unlike CTCF-mediated loops, the interaction between GLC and the HOXA locus is broad and covers regions enriched in polycomb-silenced H3K27me3 (Figure 1a, b). The GLC also acts as a boundary to an 3' upstream TAD featuring a gene desert (Figure 1a). We speculated that the GLC could function as a cis-regulatory element to the posterior HOXA genes in addition of being a TAD boundary element. CRISPR-Cas9 mediated deletion of the GLC in cord blood CD34+ HSPCs using two flanking guide RNAs yielded a heterogeneous population of $G L C$-deleted and wild-type cells (Figure 1c, d). This population of cells differentiated more rapidly in ex vivo culture conditions compared to control cells edited for an off-target deletion (Figure 1e). Furthermore, the GLC-deleted cells yielded smaller and significantly lesser number of colonies in a colony formation assay (Figure $1 \mathrm{f}, \mathrm{g}$ ). This suggests that the GLC is a non-coding region with functional role in HSPC self-renewal activity. In situ Hi$C$ revealed that partial deletion of the GLC resulted in a significant loss of interaction with the HOXA locus, particularly at the H3K27me3 enriched regions (Figure 1h). Meanwhile, the active HOXA region enriched in H3K27ac (HOXA6-HOXA10) also lost interactions with the H3K27ac marked upstream enhancers in $S K A P 2$ and $S N X 10$ region $^{9}$ (Figure 1h, i, Supplemental Figure 
S1b, c). Consistent with the loss of this active enhancer-promoter interaction, the loss of GLC also resulted in a significant decreased expression of the active HOXA7-10 genes and a modest decrease of the interacting $H O X A 11$ and $A 13$ (Figure 1j). These results suggest that the GLC does not act as a repressor of the distal HOXA genes despite its interaction with the H3K27me3 rich region of the locus. Instead, this long-range interaction serves as an activating element for the HOXA genes in HSPCs potentially by maintaining promoter-enhancer interaction for the active HOXA genes (Figure 1k).

The HOXA transcription factors are often expressed in subtypes of human acute myeloid leukemias (AML) and overexpression of the HOXA9 gene has been shown to directly transform mouse bone marrow cells ${ }^{10}$. To determine whether the GLC plays a role in maintaining the aberrant expression of HOXA genes in leukemia, we performed in situ $\mathrm{Hi}-\mathrm{C}$ on primary human leukemia blasts from patients carrying the MLL-AF9 translocation (AML-4943), the NPM1 mutation (AML-5577, AML-6527), as well as HOXA high expressing cell lines OCI-AML3, MV411 and the HOXA low expressing cell line Kasumi-1 (Figure 2a, b, Supplemental Figure S5ad) ${ }^{11,12}$. The AML blasts were mostly CD33+ CD34- (Supplemental Figure S2a-b). The highresolution $\mathrm{Hi}-\mathrm{C}$ maps were validated through identification of known genomic translocations such as $t(9: 11)$ in $A M L-4943$ blast, $t(4: 11)$ in $M V 4-11$ and $t(8: 21)$ in Kasumi-1 cells (Supplemental Figure 3a-f). We observed that the genome-wide chromosomal loop interactions were consistent in all $3 \mathrm{AML}$ blasts by aggregate peak analysis (Supplemental Figure S2c) ${ }^{13}$. However, up to $80 \%$ of grand canyon interactions were lost in primary AML blasts and almost all such interactions were lost in cell lines (Supplemental Figure S2c, d). In tandem, we performed ChIP-seq on H3K27me3 and H3K27ac histone marks to identify regions of silent and active chromatin in these cells. Overlaying Hi-C contact maps with the histone marks revealed a pattern between the $3 D$ chromatin organization and the underlying epigenome. Strong GLC to HOXA interaction was observed in the AML-4943 patient blast cells, similar to cord blood CD34+ HSPC with comparable silent chromatin states at both GLC and HOXA regions (Figure 1a, 2a, c, Supplemental Figure 1d). Additionally, entire SKAP2 gene is marked by a very broad $\mathrm{H} 3 \mathrm{~K} 27 \mathrm{ac}$ peak and forms a stripe interaction with the active HOXA genes, which is distinct from other cell types (Figure 2a, c). In in AML-5577, the site of contact at the HOXA locus shifts patient blasts to the location of matching activating H3K4me3 marks (Supplemental Figure 5a, e). This is also observed in the OCl-AML3 cell line, which is depleted of H3K27me3 at the GLC, but strong H3K27ac at both GLC and HOXA seem to demarcate this long-range interaction (Figure $2 b, d$ ). Since both AML-5577 and OCI-AML3 carry the NPM1 ${ }^{\text {w288fs }}$ mutation, these results suggest that mutant NPM1 may be associated with the structural alteration at the HOXA 
cluster. This long-range interaction was significantly weaker in MV4-11 cells, which showed no H3K27me3 and weak H3K27ac at the GLC, and a strong but narrow enrichment of H3K27ac at the active HOXA9-A10 genes (Supplemental Figure S5b, e). Meanwhile, the low HOXA expressing Kasumi-1 cell line had HOXA interaction with convergent CTCF sites downstream of the GLC (Figure 2e, Supplemental Figure S5c). This result is particularly interesting as Kasumi1 cells feature enrichment of H3K27me3 at both GLC and distal HOXA regions, yet they do not directly form a long-range loop. Taken together, these results show that the GLC-HOXA interaction occurs primarily in cells expressing high levels of HOXA genes, and this interaction is associated by co-occurring histone modifications at both loci (Figure 2f, g).

As the GLC interacts with active HOXA locus in leukemia cells, we used paired sgRNAs to delete the GLC in OCI-AML3 and MV4-11 cell lines to determine whether it possesses similar regulatory function as in HSPC (Figure 3a). The CRISPR-Cas9 mediated-deletion created a heterogeneous population of GLC-deleted and wild-type cells which were cultured up to 21 days in a growth competition assay. Comparing the two populations at days 7 and 21, the GLCdeleted cells declined over time, indicating that the non-coding region is required for the growth of both cell lines (Figure $3 \mathrm{~b}$ ). As the GLC spans a $\sim 17 \mathrm{~kb}$ region, we used a CRISPR-Cas9 saturation mutagenesis screen to identify distinct elements within this region that are crucial for cell growth (Figure 3c, Supplemental Figure S6a). We have identified that sgRNA targets within the GLC that drop-off over a period of 15 days as well as positive control (Supplemental Figure S6b). The data suggests there are smaller regions in GLC required for maintaining cell growth. Comparing the results from both OCI-AML3 and MV4-11 cells, two hot spots were identified at CTCF binding sites within the GLC (Figure 3d, e). Curiously, these two CTCF sites are nonconvergent with respect to the CTCF sites within the HOXA cluster (Supplemental Figure S4). Individual deletion of the CTCF binding sites (designated CTCF-1 and CTCF-2) in cord blood CD34+ HSPC yielded different levels of effectiveness, yet both were found to diminish HSPC self-renewal (Figure $3 \mathrm{f}, \mathrm{g}$ ). Furthermore, several HOXA genes and the transcription factor MEIS1 were downregulated following the loss of CTCF-1 (Figure 3h). Deletion of both CTCF-1 and -2 in OCl-AML3 cell line revealed that loss of either sites could not compete in growth against wild-type cells (Figure 3i). Loss of CTCF-1, being a more efficient deletion, revealed a similar loss of HOXA and MEIS1 expression in this cell line (Figure 3j). These results suggest that, despite the divergent orientation of CTCF binding sites within the GLC, these elements have a functional role in regulating the HOXA genes. Given the GLC switched to active state marked by H3K27ac in the MV4-11 and OCl-AML3 cell lines (Supplemental Figure S6d), the 
two CTCFs may play a role in maintaining the active expression of HOXA genes by stabilizing promoter-enhancer interactions.

The orientation of CTCF motifs is a key factor in the formation of TADs and chromosomal loops $^{14}$. As both CTCF-1 and -2 are in divergent orientation from the HOXA CTCF sites, we investigated whether they formed other TADs elsewhere on the chromosome. We observed a chromosomal loop interaction between CTCF-1 and -2 and upstream (telomeric) CTCF sites (Figure 4a). This spanning region includes a single long non-coding RNA and has been identified as a lamina-associated domain (LAD) in IMR90 cells (Figure $4 a)^{15}$. Additional analysis also shows that the long DNA methylation canyon is likely to be flanked by LAD regions (Supplemental Figure 7a, b). Given the features of constitutive LADs (cLAD), which include low gene density, we surmised that this region would remain as a LAD in HSPC and leukemia cells $^{16}$. Fluorescent in situ hybridization (FISH) revealed that both distal HOXA and GLC were located at the nuclear periphery and remained in proximity to each other in cord blood CD34+ HSPCs (Figure 4b). Furthermore, the GLC was found to be close to the network of Lamin B1 located at the inner nuclear membrane in OCI-AML3 cells (Figure 4c). As such, we propose a model in which the HOXA locus is spatially tethered to the nuclear periphery through its interaction with the GLC and LAD either through repressive or activating domains (Figure 4d, e).

Our data show that the HOXA gene cluster interacts with the upstream Geneless Canyon, forming a long-range chromosomal loop tethered to the nuclear periphery of HSPCs and cell lines expressing high levels of the HOXA transcription factors. While the GLC is a polycomb target in CD34+ HSPCs, we observed that it can change epigenetic states across various tissues and cell lines. This is consistent with previous works that show how polycomb targets in early development can act as enhancers in Drosophila and mouse models ${ }^{17-20}$. We have previously shown that ablation of the silencing histone marks was sufficient to disrupt the interaction between GLC and HOXA and alters gene expression at the locus in cord blood CD34+ HSPC (Zhang et al. Molecular Cell in press). Here, we observe that the loss of GLC significantly hampers the self-renewal capability of CD34+ HSPCs and stunts growth of OClAML3 leukemia cell line model. These phenotypes are accompanied by significant downregulation of key HOXA genes. Interestingly, the long-range interaction between GLC and HOXA locus is often mediated at silencing H3K27me3 regions, yet loss of this interaction does not activate the silenced distal HOXA11 to HOTTIP genes. This suggests that the interaction itself may not be altering the epigenetic state at the HOXA cluster, but rather play a role in establishing a scaffold in 3D nuclear space that promotes HOXA gene expression. This idea has also been echoed by several recent studies with the evidence that Polycomb target loci can 
act as permissive 3D genomic structure to allow the active transcription of Homeobox transcriptional factors ${ }^{7,21}$. It is well-established that transcriptionally active regions of the genome are enriched at the nuclear center while silent regions remain at the nuclear periphery ${ }^{22}$. Furthermore, LADs are mostly associated with regions of heterochromatin marked by $\mathrm{H} 3 \mathrm{~K} 9 \mathrm{me} 3^{23}$. As such, the physical proximity of active HOXA cluster to a LAD anchored by the GLC seems counterintuitive. However, recent studies have shown that nuclear pore complexes (NPC) embedded at the nuclear periphery can associate with both enhancer and polycomb enriched regions ${ }^{24,25}$. In our effort to map NPC binding on chromatin, we observed a significant enrichment of the complex at both the active distal HOXA region as well as the GLC in OCIAML3 cells (Supplemental Figure S7c). This implies that the GLC-HOXA interaction may be the $3 D$ chromatin organization signature of tethering active HOXA to nuclear pores on the nuclear periphery, promoting rapid expression of the transcription factors in HSPC and leukemias. 


\section{Figure Legend}

Figure 1. Geneless Canyon maintains active stem cell activity and HOXA gene expression in human HSPC.

a). Hi-C contact heatmap of CD34+ HSPC (upper triangle) and differentiated erythroid progenitors (lower triangles). Grey box annotates the 3 TADs around HOXA region in both cell types. b). The epigenetic state of HOXA cluster and GLC in CD34+ HSPCs. DNA methylation, H3K27me3, H3K27ac, H3K4me3 and H3K4me1 is shown. c). Scheme of using 2 guideRNAs for the deletion of GLC in CD34+ HSPCs. d). The deletion of GLC by paired guideRNA after two rounds of RNP electroporation. e). Flow cytometry plot of HSPCs after GLC deletion with CD34FITC and CD38-PE. Flowcytometry is performed after 4 days of ex vivo culture in ex vivo expansion medium. f). Colony number of CD34+ HSPCs electroporated with paired guide RNA targeting hemoglobin locus 3'HS-1 CTCF and GLC. $\mathrm{n}=3$, mean \pm s.d. is shown. g). Colony size of CD34+ HSPCs electroporated with paired guide RNA targeting 3'HS-1 and GLC. Grid size is $1 \mathrm{~cm} \times 1 \mathrm{~cm}$. h). Hi-C contact heatmap of CD34+ HSPC and GLC-deleted C34+ HSPC (upper/lower triangles, respectively) with overlay of H3K27ac and WGBS. i). Zoom-in of HOXA interactions with upstream enhancer elements. j). HOXA gene expression after the deletion of 3'HS-1 CTCF and GLC. $\mathrm{n}=3$, mean \pm s.d. is shown. $\mathbf{k}$ ). Model of 3D genomic interactions in HOXA locus in HSPC.

Figure 2. Geneless Canyon interacts with HOXA cluster in primary leukemia samples and cell lines

a). The contact heatmap in 3 TAD regions in around HOXA cluster in primary leukemia blast AML4943. AML4943 carries the MLL-AF9 translocation. Yellow shade indicates the interacting regions of GLC and corresponding HOXA regions (HOXA11 to EVX1). Blue shade indicates the active HOXA region (HOXA7-HOXA10). Grey box annotates the interaction between SKAP2 and HOXA7-10 region (horizonal); SNX10, CBX10 (Vertical). b). The contact heatmap in 3 TAD regions in around HOXA cluster in OCLI-AML3 cell lines. Yellow shade indicates the interacting regions of GLC and corresponding HOXA (HOXA7-HOTTP) regions. All Hi-C contact map is shown with the corresponding histone ChIP-seq tracks and CD34+ HSPC canyon and whole genome bisulfite sequencing data for the location of GLC.

c). The virtual 4C plot with multiple viewpoints from GLC, 5'CTCF, HOXA7-HOXA10, HOXA11HOTTIP in the CD33+ AML-4943 Blast. d). The virtual 4C plot with multiple viewpoints from GLC, 5'CTCF, HOXA7-HOXA10, HOXA11-HOTTIP in the OCI-AML3 cell line. e). The virtual 4C 
plot with multiple viewpoints from GLC, 5'CTCF, HOXA7-HOXA10, HOXA11-HOTTIP in the Kasumi-1 cell line. Yellow bars highlight the viewpoint regions and blue bars highlight the interacting regions with the specific viewpoint. $\mathbf{f}-\mathbf{g}$ ). 3D genomic interaction model of 3' TAD and HOXA cluster in AML-4943 blast (f) and OCI-AML3 cell lines $(\mathbf{g})$.

Figure 3. Geneless Canyon and CTCF sites within is essential to leukemia cell line maintenance and HOXA gene expression.

a). The scheme of competition assay between GLC deletion and WT cell lines with CRISPR/Cas9. b). The gel picture of GLC deletion and wild type band in cell competition assay for OCl-AML3 and MV4-11 cells. c). Scheme for the tilling CRISPR/Cas9 mutagenesis for GLC. d). The mapping of guide RNA drop-off and enrichment score (calculated as log10(normalized count $_{\text {day } 15} /$ normalized count dayo $_{\text {) }}$ ) in OCl-AML cells. OCL-AML3 CTCF, H3K27ac and DNA methylation is shown in parallel e). The mapping of guide RNA drop-off and enrichment score (calculated as log10(normalized count $_{\text {day } 15} /$ normalized $_{\text {count }}$ day0) $_{\text {) }}$ in MV4-11 cells. MV4-11 CTCF, H3K27ac is shown in parallel. f). Gel picture of CTCF1 and CTCF2 deletion with paired guide RNA in cord blood CD34+ HPSC. g). Serial dilution colony forming assay with Cas9, CTCF1-deletion and CTCF2-deletion CD34+ HSPCs. h). qPCR detection of active HOXA gene expression in CD34+ HSPCs, $n=3$, mean \pm s.d. is shown. i). The gel picture of CTCF-1 and CTCF-2 deletion and wild type band in cell competition assay for OCI-AML3 and MV4-11 cells. h). qPCR detection of HOXA9, HOXA10 and MEIS1A gene expression in OCI-AML cell line, $n=3$, mean $\pm s . d$. is shown.

\section{Figure 4. Geneless Canyon-HOXA interaction is located at nuclear periphery}

a). The distribution of Lamin associated domain around HOXA cluster. b). FISH image of GLC (green) and HOXA (red) in the cord blood CD34+ cells c). Immuno-FISH image of GLC (red) and LNMB (green) in OCl-AML3 cells. d-e). Model of HOXA cluster genomic locus in 3D in normal HSPC (d) and OCI-AML3 leukemia cell lines (e).

\section{Method:}

\section{RNP based CRISPR deletion of GLC region}

RNP based CRISPR deletion was performed followed by the previous protocol ${ }^{26}$. RNP complex was prepared by mixing $2 \mu \mathrm{g}$ Cas 9 and $1 \mu \mathrm{g}$ sgRNA targeting each side of region of interest. RNP complex was incubated at 37 degree for 15 minutes before the electroporation. 2$3 \times 10^{5}$ cells were used for one electroporation by NEON transfection system. CD34+ cells were 
electroporated with Buffer T under the condition "1600V, 10ms, 3 pulses". Leukemia cell lines OCI-AML3, MV4-11 and Kasumi-1 were electroporated under the condition with Buffer R under the condition "1350V, 35ms, 1 pulse". Deletion of targeted region was generally checked 12-16 hours after electroporation. Both purchased chemical modified guide RNA (Synthego) and in vitro transcribed guide RNA were used in this study. The target region sequence of guide RNA is listed below:

GLC-5'-sgRNA-target (chemical modified guide RNA) : AAAAGACACACCGGCGTG GLC-3'-sgRNA-target (chemical modified guide RNA) : TCAGGAGGAAGGAGAACC GLC-CTCF-1-sgRNA-5' (oligos for IVT):

TtaatacgactcactataGGAAAAGACACACCGGCGTGgttttagagctagaaATAGC GLC-CTCF-1-sgRNA-3' (oligos for IVT):

TtaatacgactcactataGGCTGACTCGCGGGTGGGGAgttttagagctagaaATAGC GLC-CTCF-2-sgRNA-5'(oligos for IVT):

TtaatacgactcactataGGGGGTGGAGATGGTGAGAAgtttagagctagaaATAGC GLC-CTCF-2-sgRNA-3'(oligos for IVT):

TtaatacgactcactataGGGCGGGTTTCTCCAAGGAAgttttagagctagaaATAGC 3'HS-1-CTCF-sgRNA-5' (oligos for IVT):

TtaatacgactcactataGGaGTCTTGGGATGGCTGAAGgttttagagctagaaATAGC 3'HS-1-CTCF-sgRNA-3' (oligos for IVT):

TtaatacgactcactataGGtCCAAGGCAGGACATGTGTgtttagagctagaaATAGC HBB-3' enhancer-5'-sgRNA (oligos for IVT): taatacgactcactataGGTAGGTAGATGCTAGATTCgttttagagctagaaatagc HBB-3' enhancer-3'-sgRNA (oligos for IVT): taatacgactcactataGGAGAGCTAGTTCAAACCTTgttttagagctagaaatagc

GLC-WT-F gacagaaacttcccaggatgg

GLC-WT-R gggttggtgagattagccataaa

GLC-deletion-F (same as GLC-WT-5') gacagaaacttcccaggatgg

GLC-deletion-R atgggatgagcaaatggaaatg

GLC-CTCF1-F: GACAGAAACTTCCCAGGATGG

GLC-CTCF1-R: GACCAGGGTTGGTGAGATTAG

GLC-CTCF2-F: AGTGCCAGTATGTTCTCACTTC

GLC-CTCF2-R: GTACGACCTCACGTGTTCAG

HBB-3' enhancer -F: gaCAAGGACCACTTGAGACTC

HBB-3' enhancer -R: CTGCCTATCAGAAAGTGGTGG 


\section{HSPC ex vivo expansion and colony forming assay}

CD34+ cord blood hematopoietic stem and progenitor cells were purchased from StemCell Tech. CD34+ cells were electroporated with $2 \mu \mathrm{g}$ Cas9 and $1 \mu \mathrm{g}$ guide RNA targeting 5' of GLC and $1 \mu \mathrm{g}$ guide RNA targeting 3' of GLC. CD34+ cells electroporated with $2 \mu \mathrm{g}$ Cas9 and $1 \mu \mathrm{g}$ guide RNA targeting 5' of 3'HS-1 CTCF binding site in HBB locus and $1 \mu$ g guide RNA targeting 3' of 3'HS-1 were used as the control. CD34+ were recovered overnight after thawing in ex vivo expansion medium (SFEM II, 100ng/mL SCF, TPO, FLT3L), cells were then electroporated every 16 hours for three times. The deletion of GLC was checked after three electroporation. Cells from GLC and 3'HS-1 deletion was used for flow cytometry assay with CD34 (FITC, clone 581, BioLegend) and CD38 (PE, clone HB-7, BioLegend) for the check of cell differentiation. 200 GLC deleted and 3'HS-1 deleted CD34+ cells were then plated in 6 well plate with $1 \mathrm{~mL}$ of MethoCult ${ }^{T M}$ H4034 Optimum (StemCell technology). Colonies were counted 7 days after plating and the colony pictures were taken at the same time.

\section{CRIPSR-deletion competition assay}

OCI-AML3, MV4-11 cells were electroporated with $2 \mu \mathrm{g}$ Cas9 and $1 \mu \mathrm{g}$ guide RNA targeting 5' of GLC and $1 \mu \mathrm{g}$ guide RNA targeting 3' of GLC. Cells were placed in the continuous culture. On day 14 the $20 \%$ of cultured cells were collected and digested with DirectPCR Lysis reagent (Viagen) and Proteinase K(Viagen), and on day 21, $20 \%$ of cultured cells were collected and digested. PCR were performed detecting the wild-type and deletion.

\section{Hi-C library generation for cell line and primary human AML}

OCI-AML3, MV4-11 and Kasumi-1 cell lines Hi-C libraries were constructed with Arima Hi-C kit according to the manufacturer's instruction. 1-2 million cells are used for the construction of $\mathrm{Hi}-\mathrm{C}$ libraries. Each sample was sequenced to low depth to preform quality control check with Hi-C-Pro before sequencing to high depth ${ }^{27}$.

Primary human AML samples were purchased from Stem Cell \& Xenograft Core at University of Pennsylvania from de-identified frozen mononuclear cells from AML patients. Samples were obtained from the Stem Cell and Xenograft Core of the University of Pennsylvania. Samples were obtained after IRB consent and provided to us as annotated, anonymous samples. Mononuclear cells were thawed by directly pipetting ice cold PBS $+2 \%$ BSA to frozen cells in the freezing vials under room temperature. Thawed mononuclear cells were then gradient separated with Lymphoprep (StemCell Tech). Buffy coat was then collected to get the alive cells. Cells were stained for CD33 (PE, clone WM53, BioLegend), CD34 (APC, 
clone 581, BioLegend), CD45 (FITC, clone HI30, BioLegend), and CD38 (PeCy7, clone HB-7, BioLegend) to check for immunophenotyping by flow cytometry. Mononuclear cells were then fixed with $2 \%$ Formaldehyde for 10 mins and quenched by $0.125 \mathrm{M}$ glycine for 5 mins. In situ Hi-C is performed with fixed mononuclear cells following the previous protocol ${ }^{13}$. Cells were digested by Dpnll (NEB) for 2 hours, end filling with biotin-dATP (Jena Bioscience) and then ligated overnight. Ligated DNA was isolated by Pheno-chloroform extraction and ethanol precipitation with phase lock tube (Qiagen). Isolated DNA was then sonicated to 200-600bp with Covaris E220 and enriched for biotin containing ligated fragments using streptavidin magnetic beads (ThermoFisher). Enriched biotin containing ligated fragments were then made to library using Accel-NGS 2S kit (Swift Bioscience).

\section{CRISPR tilling screening of GLC region}

20bp oligonucleotide pool with guide RNA sequence tilling GLC region was synthesized by CustomArray. Oligonucleotide pool was then cloned using Gibson assembly (NEB) into BsmBI cut lentiviral vector ipUSEPR, which contains RFP and Puro as the selection marker. 293T cells were then transfected with pooled guide RNA libraries and helper plasmid pSMD2.G and pSAX2 for the production of lentivirus carrying pooled guide RNA. Unconcentrated medium is collected for the lentivirus carrying guide RNA pool. OCl-AML3 cells and MV4-11 cells were then transduced with titrated pooled guide RNA lentivirus. The titration that gave transduction efficiency between 20 and $30 \%$ was selected for large scale transduction, to ensure the M.O.I is lower than 1.

OCI-AML3 and MV4-11 cells were transduced with pLenti-Cas9-Blast lentivirus (Addgene: 52962). Transduced cells were maintained in $10 \mu \mathrm{g} / \mathrm{mL}$ blasticidin (ThermoFisher). Single clones of the OCl-AML3-Cas9 and MV4-11-Cas9 cells were selected and maintained in $10 \mu \mathrm{g} / \mathrm{mL}$ blasticidin. To perform large scale transduction, 10 million OCI-AML3-Cas9 and MV4-11-Cas9 cells were transduced with lentivirus carrying pooled guide RNA. 2 days after transduction, $1 \mu \mathrm{g} / \mathrm{mL}$ puromycin (ThermoFisher) is added to cell culture. One day after puromycin selection, dead cells were removed by gradient density separation with Lymphoprep (StemCell Tech). Live cells were then kept under puromycin and blasticidin for 15 days. Cells are sampled at day 0 and day 15 of the passage to survey the GLC guide RNA pool. Genomic DNA was extracted from cells in culture at day 0 and day15. Guide RNA pool was amplified with the following primer F- CTTGTGGAAAGGACGAAACACCG, R- CCTAGGAACAGCGGTTTAAAAAAGC. Illumina sequencing indexes were added by PCR amplification and indexed libraries were sequenced on HiSeq2000 by Fulgent. Guide RNA with specificity score less than 35 was filtered out, and the 
remaining guide RNA sequence was aligned to GLC genomic location. The abundance of certain guide RNA was normalized in day 0 and day 15 library. The drop-off and enrichment score was calculated as $\log 10\left(\frac{\text { normalized day } 15 \text { count }}{\text { normalized day } 0 \text { count }}\right)$

\section{ChIP-seq}

ChIP in OCI-AML3 and MV4-11 cell lines are performed with previously described ChIPmentation protocol ${ }^{28}$. One million cells were fixed with $1 \%$ formaldehyde and sonicated with Covaris E220. Sonicated chromatin were then incubated with antibody against H3K27me3(Cell Signaling, \#9733), Nuclear Pore Complex (Abcam, Mab414, ab24609) and CTCF (Abcam, \#ab70303) overnight. Protein A/G beads (ThermoFisher) were added to the sonicated chromatin for 2 hours. After washes with low-salt, high-salt and LiCl wash buffer. Following washes, beads were tagmented by Illumina tagmentation enzyme mix for 10 mins at $37^{\circ} \mathrm{C}$. After the tagmentation reaction stopped and the wash of beads, pull-downed DNA was de-crosslinked with Proteinase $\mathrm{K}$ (Zymo Research) at $55^{\circ} \mathrm{C}$ for 3 hours. Pull-downed DNA was extracted by DNA clean and concentration kit (Zymo Research). PCR amplification was performed, and libraries were sequenced with Illumia NextSeq Platform.

\section{FISH imaging}

FISH probes were purchased from Empire Genomics. The BAC clone RP11-1025G19 was used for the labelling of HOXA locus (HOXA-AS3 to EVX1). The BAC clone RP11-598H18 was used for the labelling of GLC locus. FISH was performed with the modification of protocol used previously ${ }^{29}$. Cells were fixed with $4 \%$ paraformaldehyde (Sigma-Aldrich) and spread on Superfrost Plus microslides (VWR). Coverslips were incubated in $20 \%$ glycerol in PBS for one hour at room temperate, dipped in liquid nitrogen for 6 time, washed three times with PBST in coplin jar for 5 minutes each, rinsed and incubated on ice with $0.1 \mathrm{M} \mathrm{HCl} / 0.7 \%$ Triton X-100/2x saline-sodium citrate (SSC) for $10 \mathrm{~min}$. The coverslips were then washed with $2 \times$ SSC for 3 times in coplin jar for 5 minutes each. $2 \mu \mathrm{L}$ FISH probes were then added to coverslips. Coverslips are then mounted and sealed with rubber cement. Samples were denatured on Visys HYBRITE heat block at $76^{\circ} \mathrm{C}$ for 3 min and then hybridized $18-72 \mathrm{~h}$ on Visys HYBRITE heat block at $37^{\circ} \mathrm{C}$.

Coverslips were washed at $37^{\circ} \mathrm{C}$ for 5 min three times in $2 \times \mathrm{SSC}$ and then at $60^{\circ} \mathrm{C}$ for 5 min three times in $0.1 \times$ SSC and then at room temperature in $4 \times$ SSC for $5 \mathrm{~min}$. Coverslips were mounted in DAPI-containing antifade mounting media. The image was taken by Nikon A1plusRSI confocal microscope. 


\section{Immuno-FISH imaging}

For anti-LaminB Immuno-FISH, cells were firstly fixed in 3\% paraformaldehyde in PBS for 10 min at room temperature and washed in PBST for 5 min 3 times. Cells were then permeabilized with $0.1 \%$ Triton-X at room temperature for 10 mins, then washed in PBST for 5 min 3 times. Cells were then incubated with rabbit anti-LaminB antibodies diluted 1:1,000 in blocking buffer overnight at $4^{\circ} \mathrm{C}$, washed with PBST 5 min for three times at room temperature, incubated for 1 hour at RT with Alexa488-labeled secondary goat anti-rabbit IgG (ThermoFisher.). Cells were then proceeded to FISH protocol mentioned above for washes and pretreatment. After 2XSSC washes, Coverslips were incubated at $2 \times S S C$ overnight at $37^{\circ} \mathrm{C}$ before the FISH probes were added. Coverslips were then proceeded to FISH protocol mentioned above for the FISH hybridization.

\section{Loop calling}

Hi-C Computational Unbiased Peak Search is used to call loops from Hi-C contact maps of CD34+ and GLC-deleted cell lines. The loops are called at $25 \mathrm{~kb}$ resolution. Other parameters are set as default values, namely false discovery rate $(F D R)=0.1$, window size $=3$ and peakmerging threshold $=50 \mathrm{~kb}$.

HICCUPS compares the contact reads of each pixel with its four nearby regions named horizontal, vertical, donut and lower-left, which correspond to the horizontal / vertical lines crossing at the pixel, the doughnut-shaped surrounding region and the lower-left quarter of the ring, respectively. Then a Benjamini-Hochberg FDR control procedure is applied to filter the significantly enriched pixels. Only if the four regions' q-values are all below the FDR threshold can a region be identified as a loop. 


\section{Data access:}

All processed data for visualization can be accessed at the following URL https://www.dropbox.com/sh/6wu95419kfwaenj/AADMDgwAB4RK6GqIFH0meeUZa?dl=0

Public data used in this study:

GEO accession number GSM3024909 for OCI-AML3 H3K27ac ChIP-seq; GEO accession number GSM1534446 for Kasumi-1 H3K27me3 ChIP-seq; GEO accession number GSM2212053 for Kasumi-1 H3K27ac ChIP-seq; GEO accession number GSM1525022 for OCIAML3 whole genome bisulfite sequencing; GEO accession number GSM486704 for CD34+ HSPC H3K27me3; GEO accession number GSM916052 for CD34+ HSPC whole genome bisulfite sequencing; GEO accession number GSM772870 for CD34+ HSPC H3K27ac.

\section{Author Contribution}

X.Q.D.W and X.Z conceptualized the idea and performed experiments, analyzed the data. W.Z and P.H analyzed Hi-C data. H.G generated Hi-C and ChIP-seq libraries. Y.L performed FISH and immuno-FISH staining. Y.L and C.C designed guideRNA library targeting GLC. F.F and J.L analyzed and quantified the 3D genomic interaction data. G.P provide advice and jointly supervise the project with X.Z. All authors participated in the writing and editing of the manuscript.

\section{Acknowledgement}

We acknowledge Drs. Margaret Goodell and Mira Jeong for the initial contribution to the project. We thank for Drs. Yun Huang, Stanley Lee and Yali Dou for the help of reviewing the manuscript. X.Z is supported by VARI fellowship, ASH scholar award and is an EvansMDS Young Investigator. 


\section{References}

1. Alharbi, R.A., Pettengell, R., Pandha, H.S. \& Morgan, R. The role of HOX genes in normal hematopoiesis and acute leukemia. Leukemia 27, 1000-8 (2013).

2. Abramovich, C., Pineault, N., Ohta, H. \& Humphries, R.K. Hox genes: from leukemia to hematopoietic stem cell expansion. Ann N Y Acad Sci 1044, 109-16 (2005).

3. Sauvageau, G. et al. Differential expression of homeobox genes in functionally distinct CD34+ subpopulations of human bone marrow cells. Proc Natl Acad Sci U S A 91, 12223-7 (1994).

4. Gough, S.M., Slape, C.I. \& Aplan, P.D. NUP98 gene fusions and hematopoietic malignancies: common themes and new biologic insights. Blood 118, 6247-57 (2011).

5. Krivtsov, A.V. \& Armstrong, S.A. MLL translocations, histone modifications and leukaemia stem-cell development. Nat Rev Cancer 7, 823-33 (2007).

6. Berlivet, S. et al. Clustering of tissue-specific sub-TADs accompanies the regulation of HoxA genes in developing limbs. PLoS Genet 9, e1004018 (2013).

7. Gentile, C. et al. PRC2-Associated Chromatin Contacts in the Developing Limb Reveal a Possible Mechanism for the Atypical Role of PRC2 in HoxA Gene Expression. Dev Cell 50, 184-196 e4 (2019).

8. Joeng, M. et al. A Cell Type-Specific Class of Chromatin Loops Anchored at Large DNA Methylation Nadirs. bioRxiv, 212928 (2017).

9. Castillejo-Lopez, C. et al. Detailed Functional Characterization of a Waist-Hip Ratio Locus in 7p15.2 Defines an Enhancer Controlling Adipocyte Differentiation. iScience 20, 42-59 (2019).

10. Kroon, E. et al. Hoxa9 transforms primary bone marrow cells through specific collaboration with Meis1a but not Pbx1b. Embo J 17, 3714-25 (1998).

11. Drabkin, H.A. et al. Quantitative HOX expression in chromosomally defined subsets of acute myelogenous leukemia. Leukemia 16, 186-95 (2002).

12. Spencer, D.H. et al. Epigenomic analysis of the HOX gene loci reveals mechanisms that may control canonical expression patterns in AML and normal hematopoietic cells. Leukemia 29, 1279-89 (2015).

13. Rao, S.S. et al. A 3D map of the human genome at kilobase resolution reveals principles of chromatin looping. Cell 159, 1665-80 (2014).

14. de Wit, E. et al. CTCF Binding Polarity Determines Chromatin Looping. Mol Cell 60, 676-84 (2015).

15. Sadaie, M. et al. Redistribution of the Lamin B1 genomic binding profile affects rearrangement of heterochromatic domains and SAHF formation during senescence. Genes Dev 27, 1800-8 (2013).

16. Meuleman, W. et al. Constitutive nuclear lamina-genome interactions are highly conserved and associated with A/T-rich sequence. Genome Res 23, 270-80 (2013).

17. Taberlay, P.C. et al. Polycomb-repressed genes have permissive enhancers that initiate reprogramming. Cell 147, 1283-94 (2011). 
18. Chan, H.L. et al. Polycomb complexes associate with enhancers and promote oncogenic transcriptional programs in cancer through multiple mechanisms. Nat Commun 9, 3377 (2018).

19. Erceg, J. et al. Dual functionality of cis-regulatory elements as developmental enhancers and Polycomb response elements. Genes Dev 31, 590-602 (2017).

20. Ngan, C.Y. et al. Chromatin interaction analyses elucidate the roles of PRC2bound silencers in mouse development. Nat Genet 52, 264-272 (2020).

21. Cruz-Molina, S. et al. PRC2 Facilitates the Regulatory Topology Required for Poised Enhancer Function during Pluripotent Stem Cell Differentiation. Cell Stem Cell 20, 689-705 e9 (2017).

22. Takizawa, T., Meaburn, K.J. \& Misteli, T. The meaning of gene positioning. Cell 135, 9-13 (2008).

23. van Steensel, B. \& Belmont, A.S. Lamina-Associated Domains: Links with Chromosome Architecture, Heterochromatin, and Gene Repression. Cell 169, 780-791 (2017).

24. Scholz, B.A. et al. WNT signaling and AHCTF1 promote oncogenic MYC expression through super-enhancer-mediated gene gating. Nat Genet 51, 17231731 (2019).

25. Gozalo, A. et al. Core Components of the Nuclear Pore Bind Distinct States of Chromatin and Contribute to Polycomb Repression. Mol Cell 77, 67-81 e7 (2020).

26. Gundry, M.C. et al. Highly Efficient Genome Editing of Murine and Human Hematopoietic Progenitor Cells by CRISPR/Cas9. Cell Rep 17, 1453-1461 (2016).

27. Servant, N. et al. HiC-Pro: an optimized and flexible pipeline for $\mathrm{Hi}-\mathrm{C}$ data processing. Genome Biol 16, 259 (2015).

28. Schmidl, C., Rendeiro, A.F., Sheffield, N.C. \& Bock, C. ChIPmentation: fast, robust, low-input ChIP-seq for histones and transcription factors. Nat Methods 12, 963-965 (2015).

29. Chen, Y. et al. Mapping 3D genome organization relative to nuclear compartments using TSA-Seq as a cytological ruler. J Cell Biol 217, 4025-4048 (2018). 


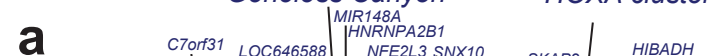

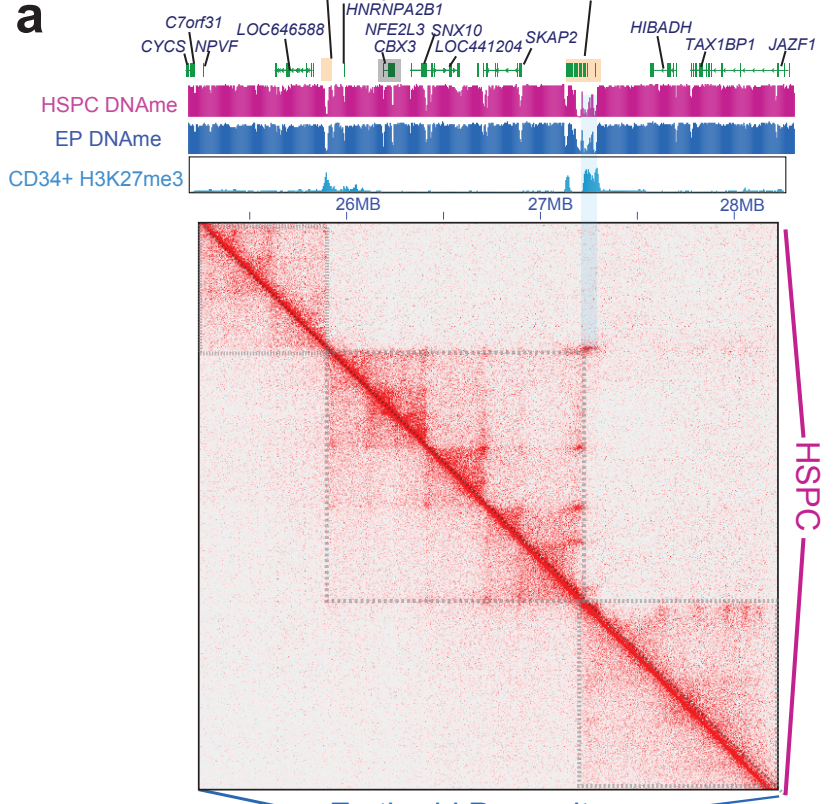

b

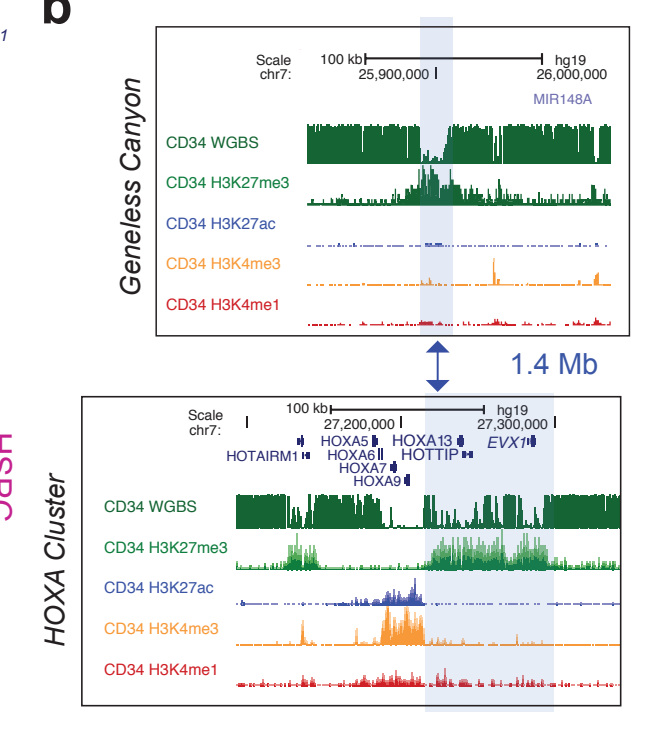

c

sgRNA-Geneless Canyon-1

+ sgRNA-Geneless Canyon-2

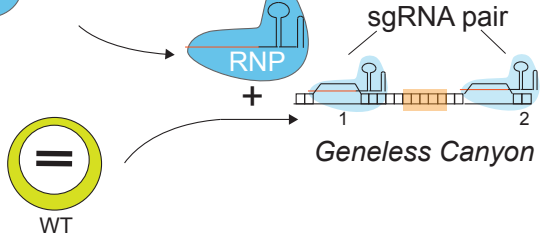

$\approx$

1. Ex vivo expression culture

$\longrightarrow$ 2. Colony formation

3. Gene expression

d
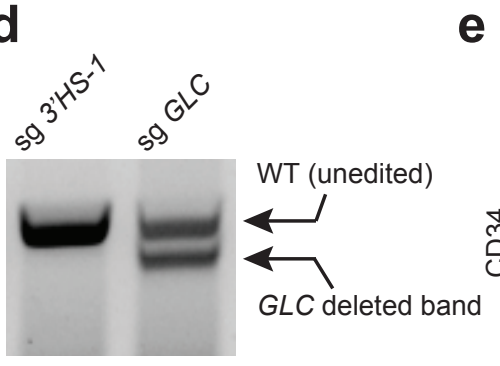

GLC SNX10 SKAP2 HOXA7-10
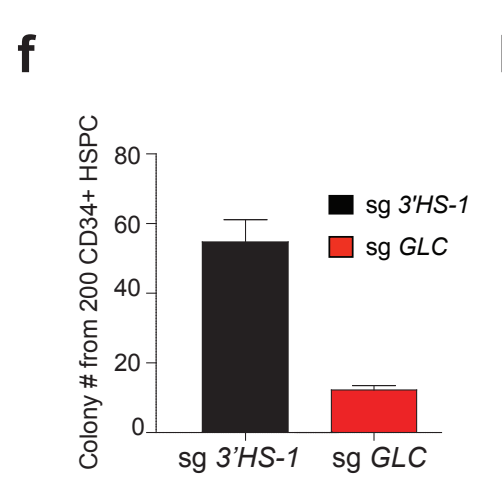

h RefSeq Genes H|t

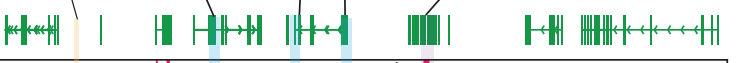
CD34+ WGBS CD $34+$ H3K27me3
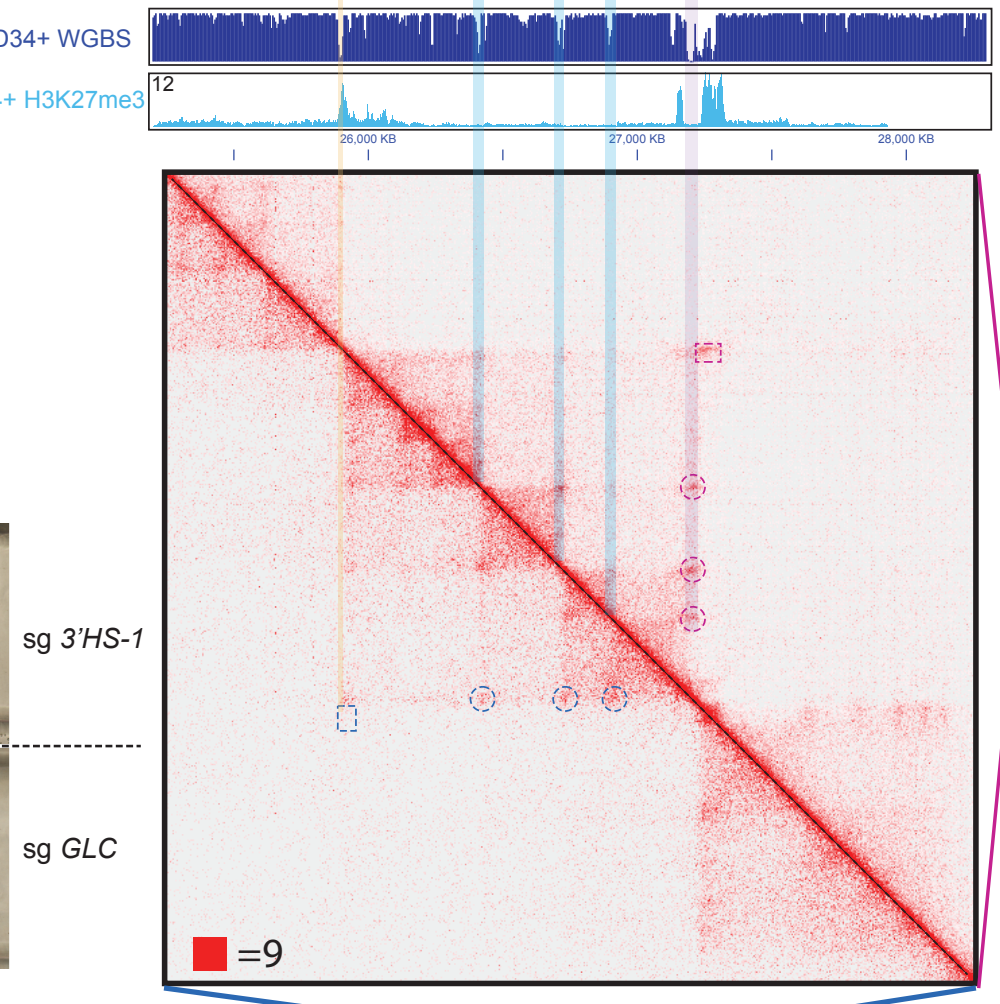

Wang et al. Figure 1 g

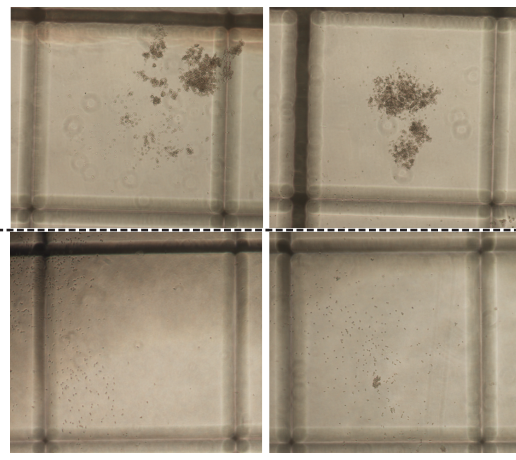

HSPC GLC Deletion

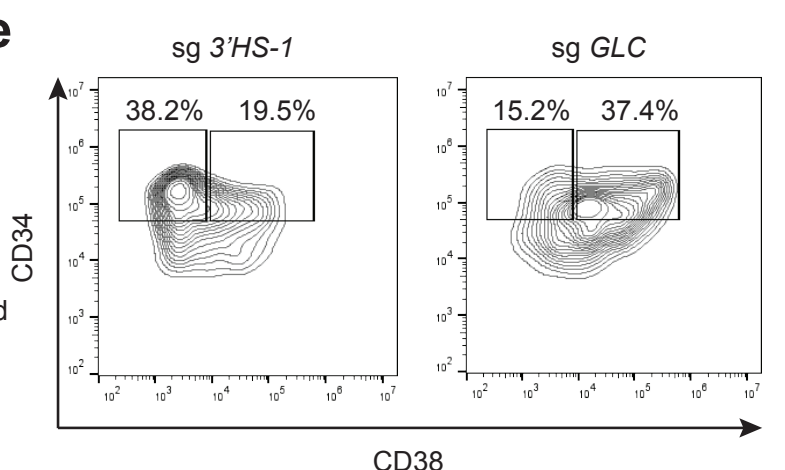

j

CD38

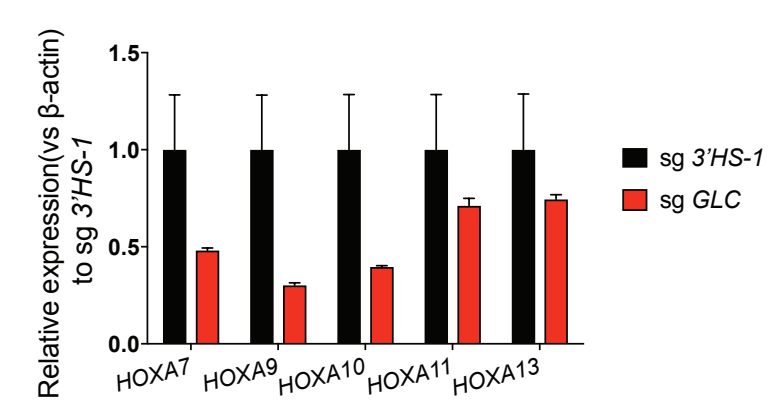
HSPC GLC i. Loop3 i, i.: Loop2 is: in: Loop1 is:

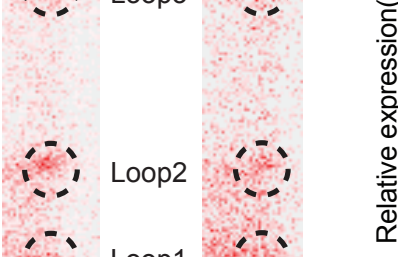

视
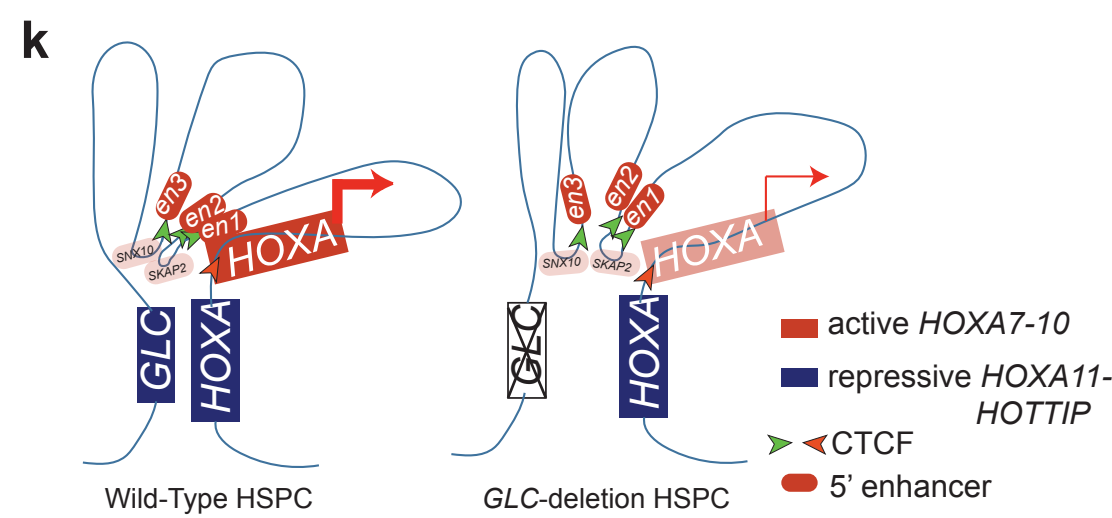
AML-4943 MLL-AF9 (CD33+)

\section{a}

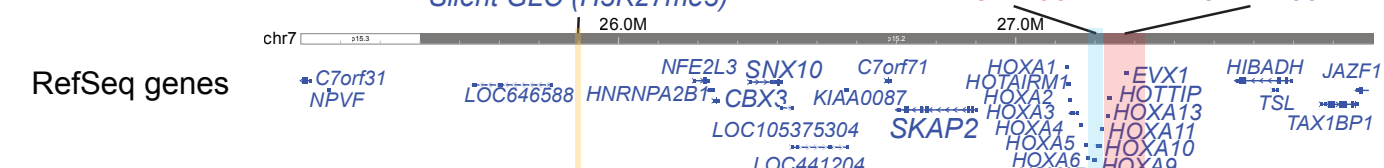

AML-4943 H3K27ac

AMLL4943 H3K27me3

CD34 canyon

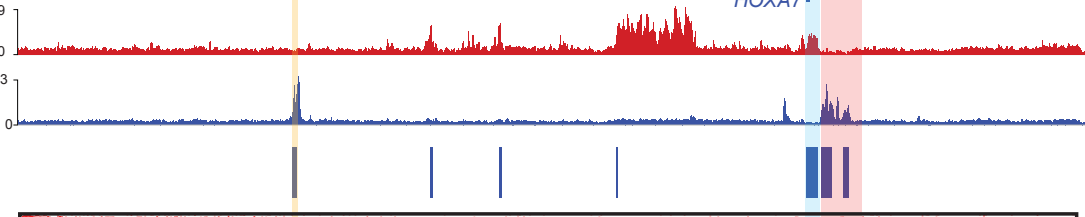

.

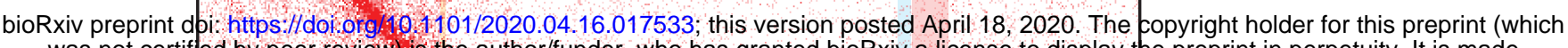
was not certified by peek review, is the authorfunder, who has granted blohxw alcense to display tre preprint in perpeturty. It is made

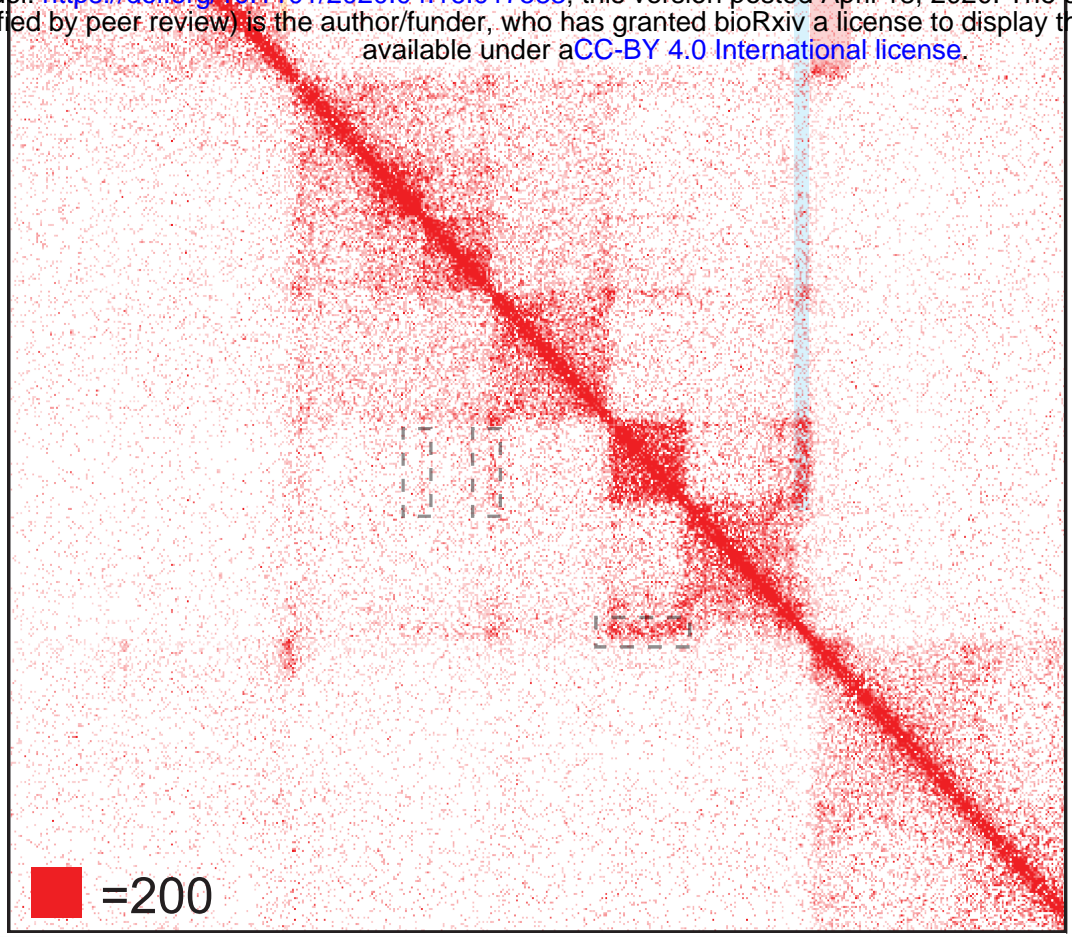

C

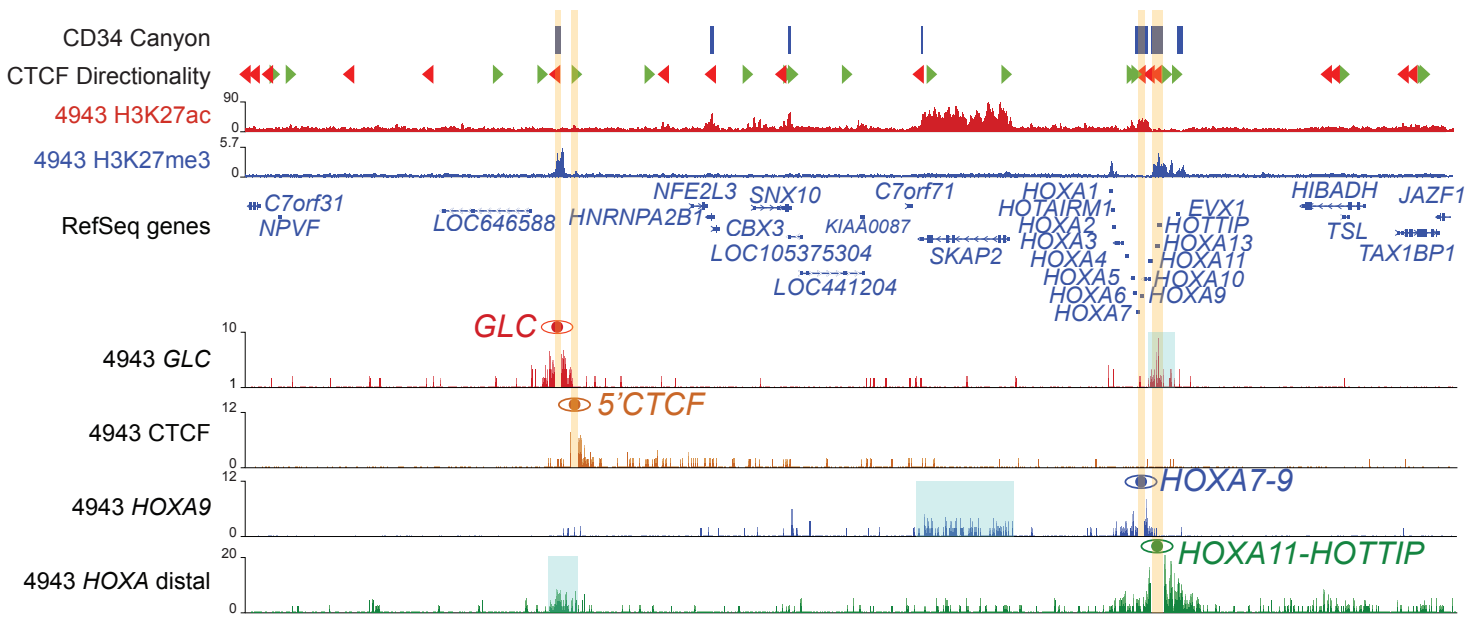

e

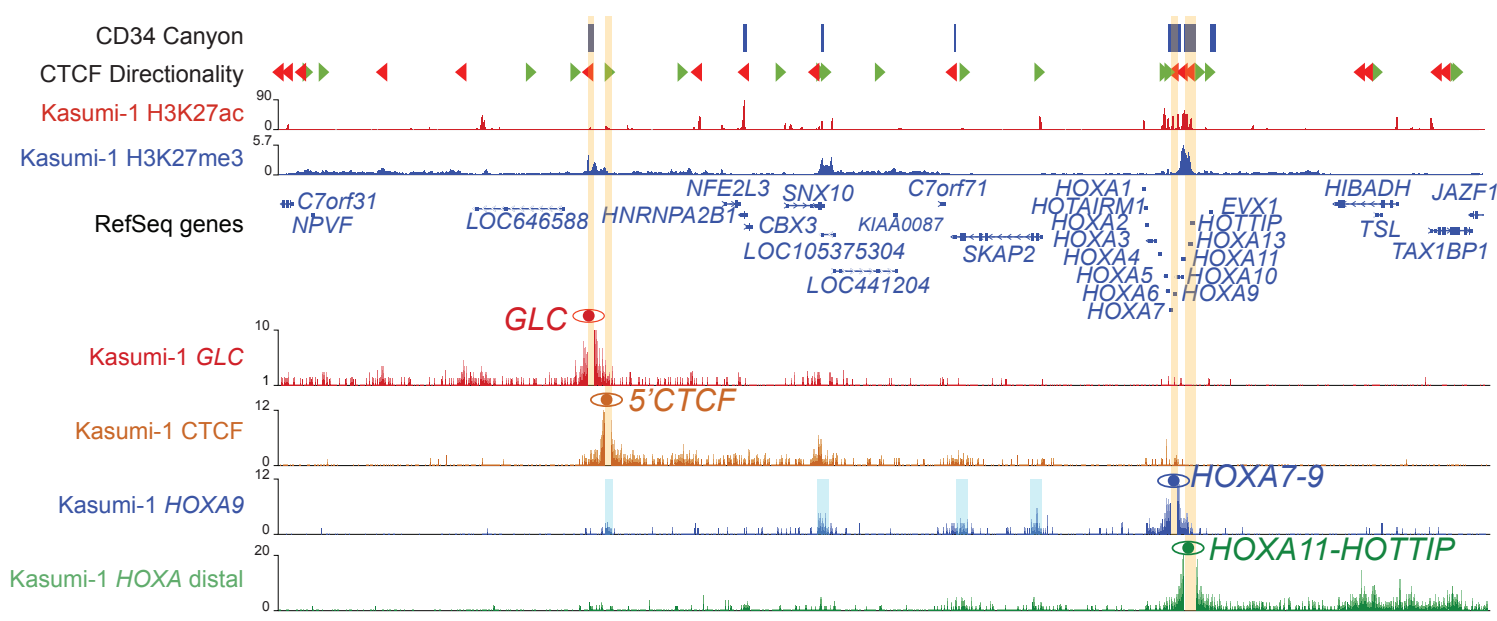

d

OCI-AML3
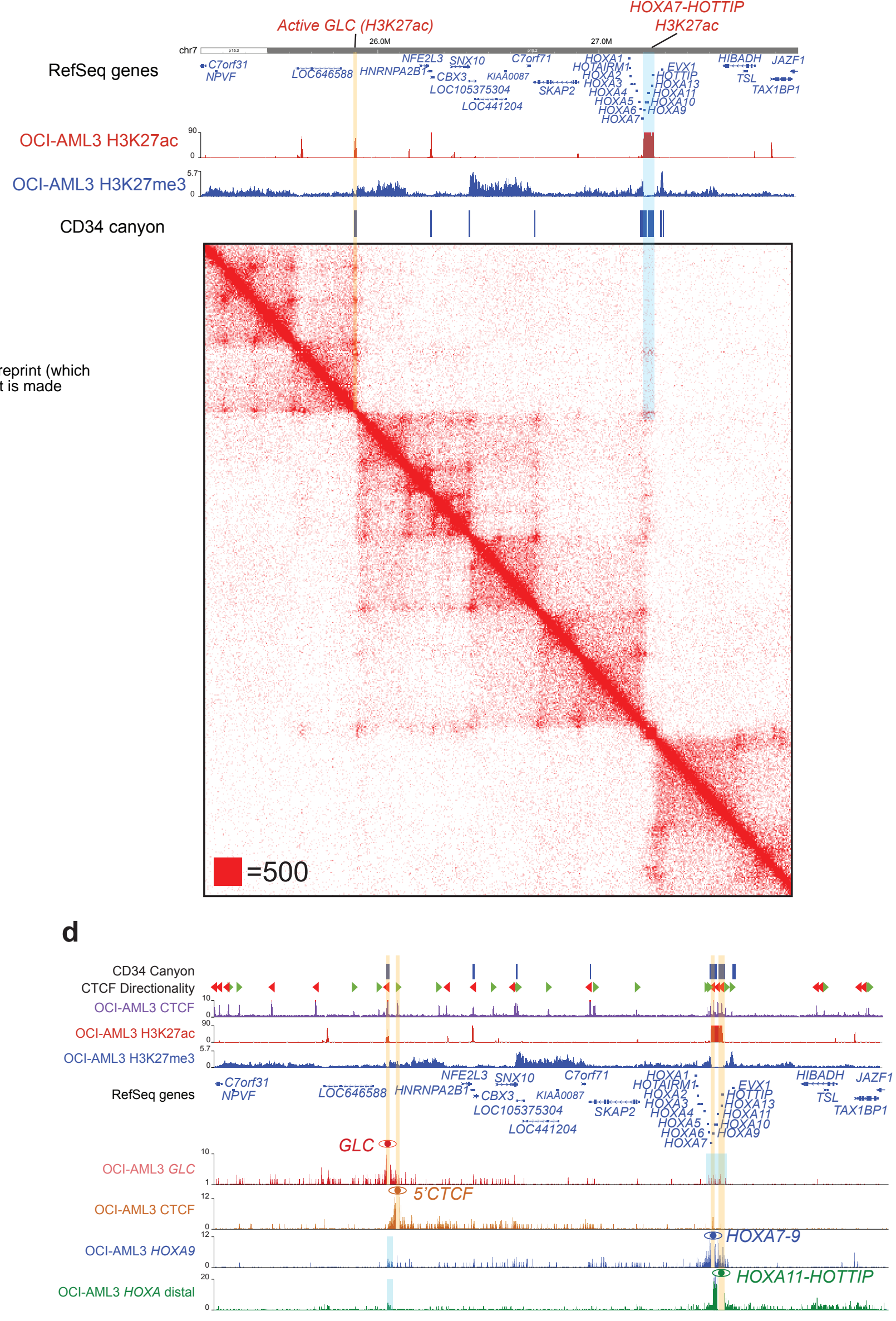

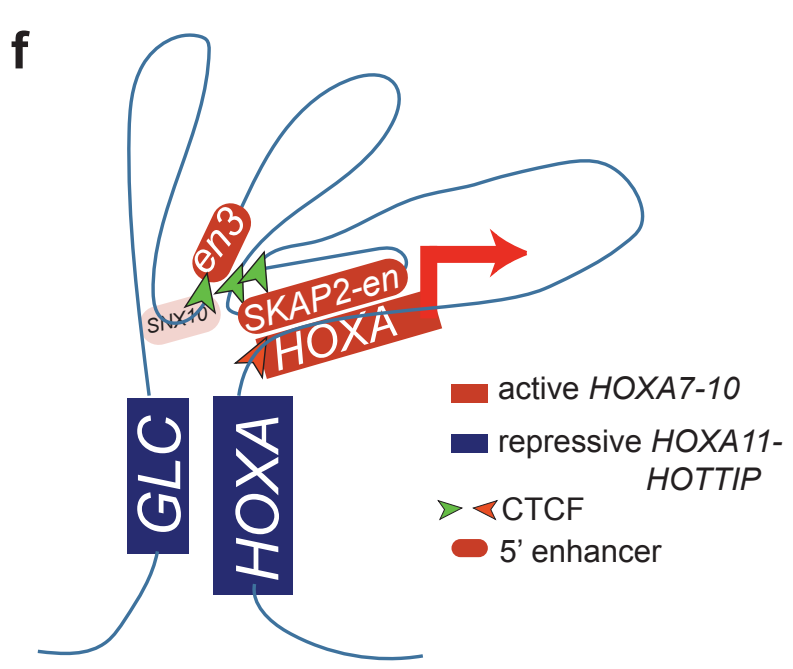

AML-4943 Blast

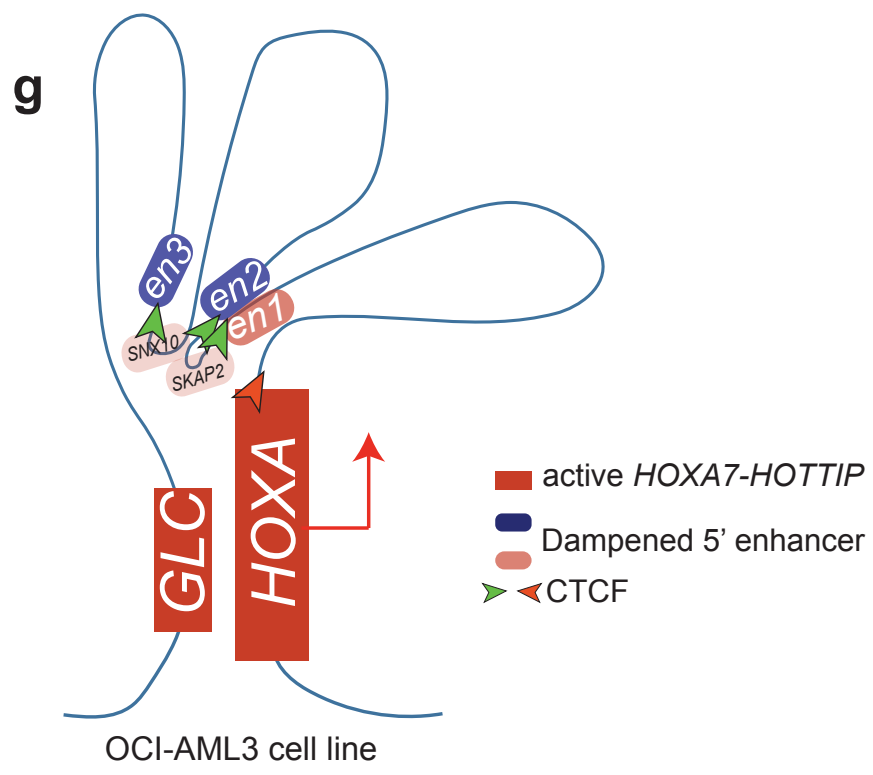

Wang et al. Figure 2 
a

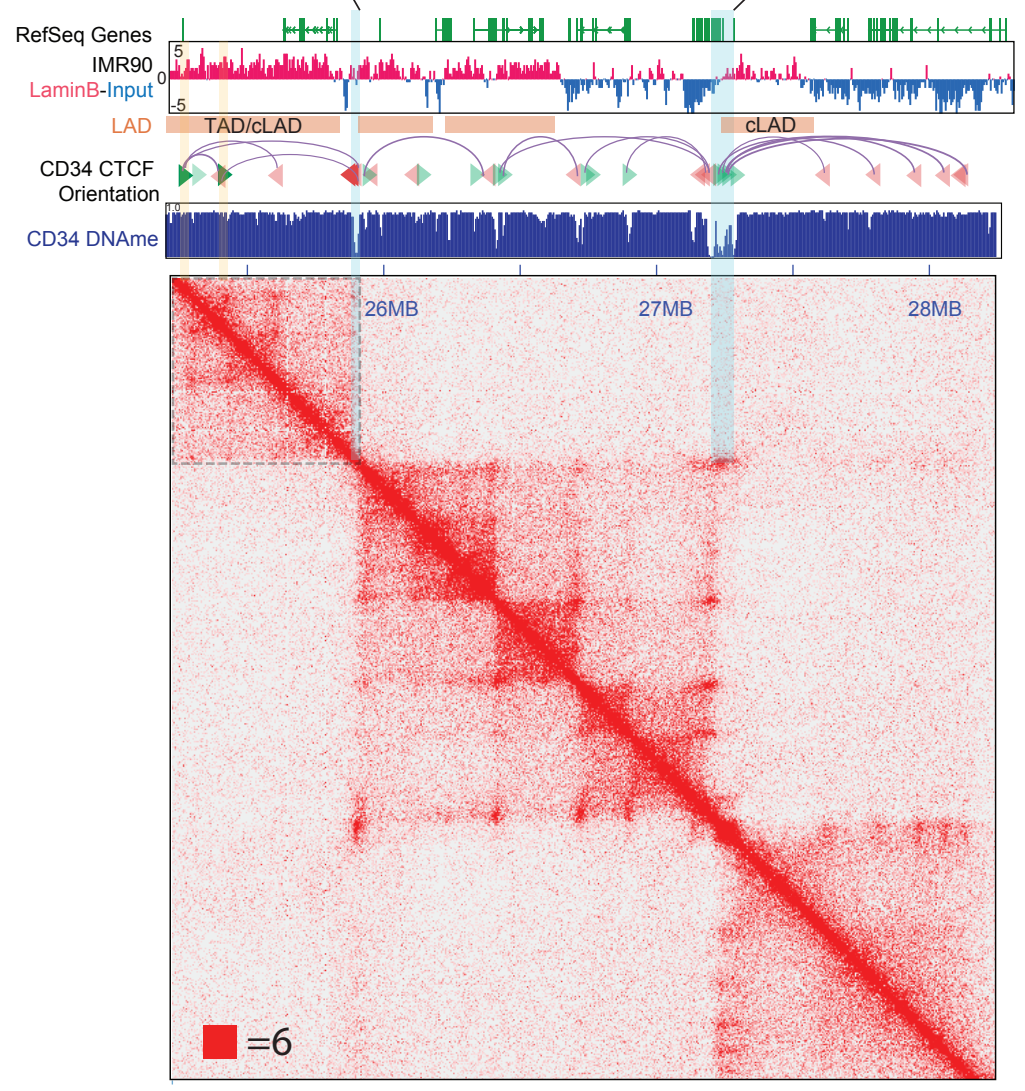

d

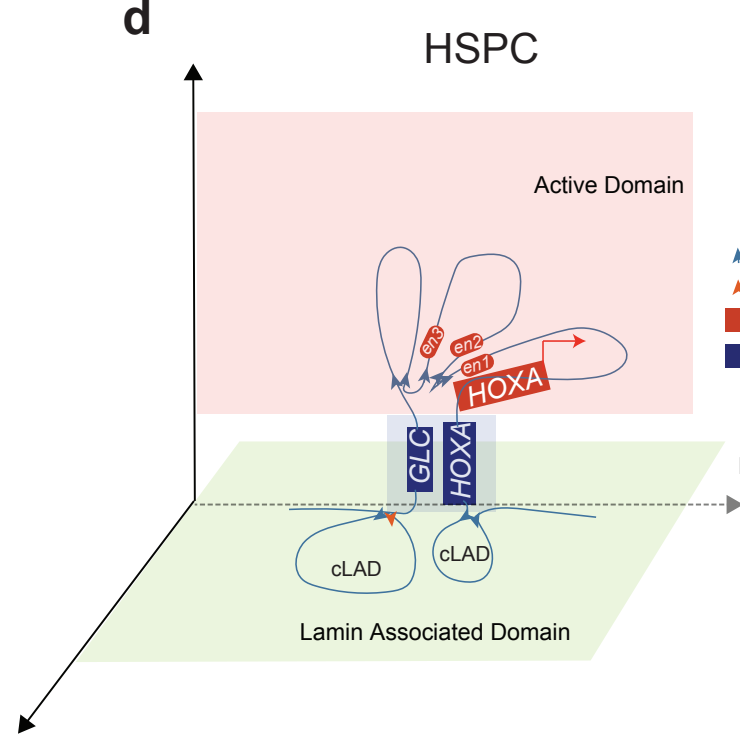

b

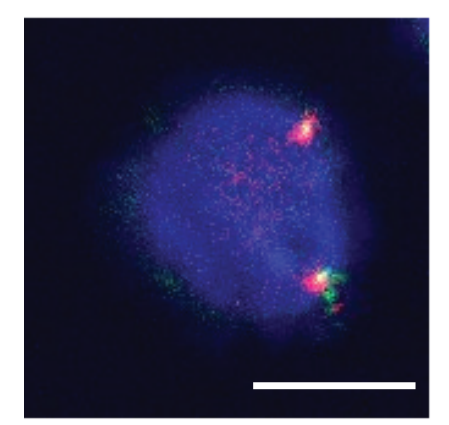

C OCl-AML3 (Geneless Canyon \& LaminB)

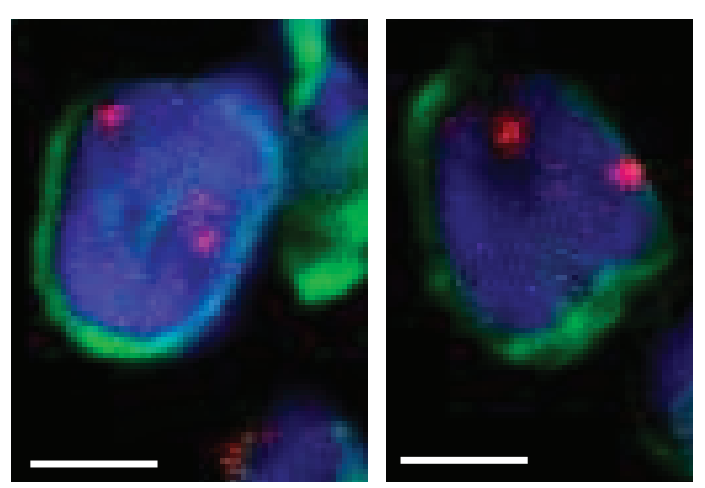

e

OCI-AML3 cell line

Weak Polycomb Domain + active transcription

A CTCF

A Border-CTCF

Active domian (H3K27ac)

Repressive (H3K27me3)

Polycomb Domain

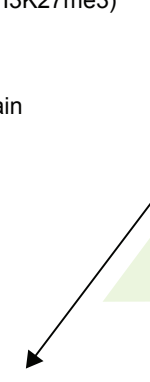

A CTCF

A Border-CTCF

Active domian (H3K27ac)

Repressive (H3K27me3)

Wang et al. Figure 4 